\title{
Transport by intermittent convection in the boundary of the DIII-D tokamak
}

\author{
J. A. Boedo, ${ }^{\text {a) }}$ D. Rudakov, R. Moyer, S. Krasheninnikov, D. Whyte, G. McKee, ${ }^{\text {b) }}$ \\ G. Tynan, M. Schaffer, ${ }^{c)}$ P. Stangeby, ${ }^{d)}$ P. West, ${ }^{d)}$ S. Allen, $\left.{ }^{e}\right)$ T. Evans, ${ }^{d)}$ R. Fonck, ${ }^{\text {b) }}$ \\ E. Hollmann, A. Leonard, ${ }^{c}$ A. Mahdavi, ${ }^{d}{ }^{d}$ G. Porter, ${ }^{e)}$ M. Tillack, and G. Antar \\ University of California, San Diego, Energy Research Center, La Jolla, California 92093
}

(Received 23 February 2001; accepted 8 August 2001)

\begin{abstract}
Intermittent plasma objects (IPOs) featuring higher pressure than the surrounding plasma, and responsible for $\sim 50 \%$ of the $E \times B_{T}$ radial transport, are observed in the scrape off layer (SOL) and edge of the DIII-D tokamak [J. Watkins et al., Rev. Sci. Instrum. 63, 4728 (1992)]. Conditional averaging reveals that the IPOs, produced at a rate of $\sim 3 \times 10^{3} \mathrm{~s}^{-1}$, are positively charged and also polarized, featuring poloidal electric fields of up to $4000 \mathrm{~V} / \mathrm{m}$. The IPOs move poloidally at speeds of up to $5000 \mathrm{~m} / \mathrm{s}$ and radially with $\mathbf{E} \times \mathbf{B}_{T} / B^{2}$ velocities of $\sim 2600 \mathrm{~m} / \mathrm{s}$ near the last closed flux surface (LCFS), and $\sim 330 \mathrm{~m} / \mathrm{s}$ near the wall. The IPOs slow down as they shrink in radial size from $4 \mathrm{~cm}$ at the LCFS to $0.5 \mathrm{~cm}$ near the wall. The IPOs appear in the SOL of both L and $\mathrm{H}$ mode discharges and are responsible for nearly $50 \%$ of the SOL radial $E \times B$ transport at all radii; however, they are highly reduced in absolute amplitude in H-mode conditions. (C) 2001 American Institute of Physics. [DOI: 10.1063/1.1406940]
\end{abstract}

\section{INTRODUCTION}

A magnetic divertor ${ }^{1}$ in a tokamak provides heat and particle exhaust and shields the main plasma from impurity contamination. Heat and particles are transported from the plasma core to the edge and scrape-off layer (SOL) plasma, where they are, in turn, conducted and convected to the divertor, which is optimized to reduce structural damage and impurity release. The balance between parallel and perpendicular transport results in a SOL which is thin compared to its length ${ }^{1}$ and the observed SOL profiles are mostly exponential ${ }^{2}$ with short $(1-3 \mathrm{~cm})$ decay lengths. However, there is ample ${ }^{3-5}$ evidence that the SOL profiles are sometimes wider than usual and nonexponential and flat far away from the last closed flux surface (LCFS), suggesting that perpendicular transport in these conditions can be larger than expected.

A candidate for the additional edge/SOL transport, intermittency in the fluctuations corresponding to a significant number of events above the standard deviation, has been extensively documented in linear devices, ${ }^{6,7}$ stellarators ${ }^{8}$ and tokamaks $^{9-11}$ and its statistical properties examined ${ }^{12}$ via the probability distribution function ${ }^{13}$ (PDF). The essential result is that the fluctuations depart from a Gaussian distribution, featuring skewness ${ }^{13}$ and kurtosis ${ }^{13}$ that correspond to the presence of a significant number of events above the standard deviation. Furthermore, it has been proven in tokamaks (JET) and stellarators ${ }^{14}$ (ATF, W7-AS) that the statistical properties of the fluctuations are alike and near-Gaussian in the shear layer while deviating from Gaussian into the edge or the SOL. ${ }^{14}$ The intermittency has been characterized using

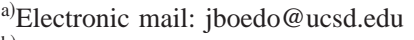

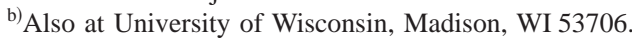

c) Also at General Atomics, San Diego, CA.

d) Also at University of Toronto, Toronto, Canada.

e) Also at Lawrence Livermore National Laboratory, Livermore, CA.
}

conditional averaging tools in both linear devices ${ }^{7,6}$ and tokamaks, ${ }^{15}$ with the result that structures propagating radially are responsible for much of the radial transport. Recent work in the CASTOR tokamak, ${ }^{16}$ using conditional averaging, reported intermittent coherent structures propagating radially and poloidally in the SOL. Comparative work at the devices TJ-I and TJ-IU concluded that intermittency bears a substantial part of the transport and, most importantly, that the various properties of the intermittency (power spectra, PDF moments, etc.) are self-similar in tokamaks and torsatrons. Recent work in the JET tokamak ${ }^{17}$ focused on the power spectra of the fluctuations, demonstrating that decay as $1 / f$ was localized in the spectral region where intermittent (or bursty) transport is dominant and interpreting the result as an indication of closeness to instability thresholds. Therefore, the universality of intermittency and the concomitant fast convective transport has been established ${ }^{6,18,19,8}$ for long. Additionally, there is an extensive body of work imaging the edge of various devices with fast cameras ${ }^{20,21}$ which show that moving plasma filaments exist in the edge/SOL. Thus, work over the years has accumulated overwhelming evidence that moving, intermittent coherent structures are convected in the edge/SOL of magnetically confined plasmas carrying a large amount of the total transport.

This article presents results from various diagnostics, probes in particular, demonstrating that fast, intermittent, convective transport perpendicular to $\mathbf{B}$ can be present in the DIII-D tokamak, accompanied by the usual non-Gaussian characteristics in the fluctuations. It is also shown that this intermittent transport is responsible for nearly $50 \%$ of the transport in both L- and H-mode conditions. Although the source of the intermittency is not addressed in this article, the point made is that the intermittent transport can change the particle and heat load distribution, and consequently the recycling, at the walls. Furthermore, the far SOL profiles are found to be flat, questioning the role of diffusion and sup- 


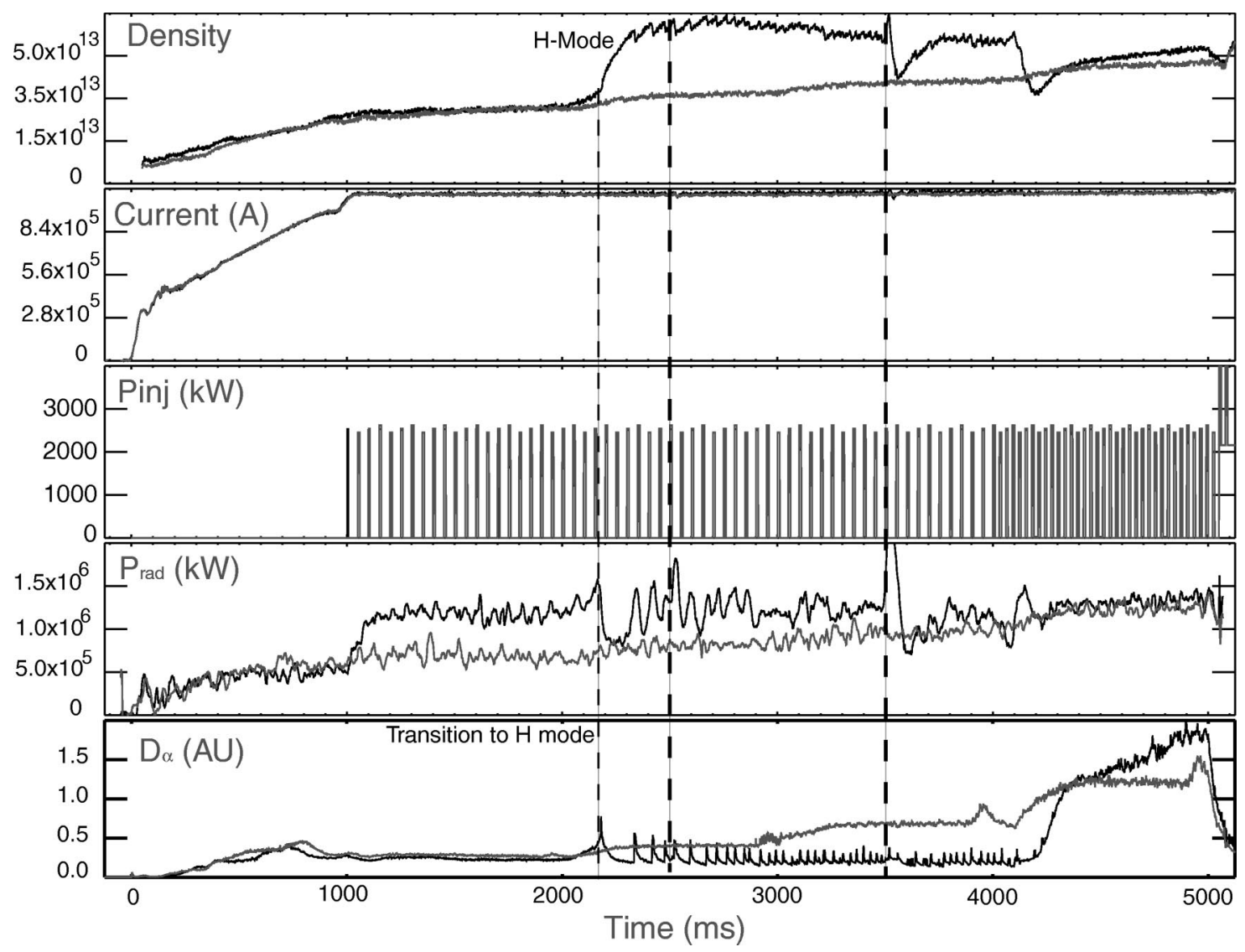

FIG. 1. Time evolution of two discharges in DIII-D showing, from top to bottom, density, plasma current, injected power, radiated power and $\mathrm{D}_{\alpha}$ signals. The transition to H-mode occurs at $2.15 \mathrm{~s}$ in one of the discharges. The probe is inserted at the times marked by the two thick vertical lines.

porting convection as the alternative transport mechanism. These findings have multiple consequences for future fusion reactor design and thus provide a strong motivation to further investigate the conditions under which large cross-field convective transport exists in the SOL and its relation to intermittency.

\section{EXPERIMENTAL SETUP}

The experiments were carried out on the DIII-D tokamak $^{22}$ for a variety of discharges with plasma current $I_{p}=1.0 \mathrm{MA}$ and toroidal field of $B_{T}=2.0 \mathrm{~T}$ at major radius, $R=1.7 \mathrm{~m}$. For completeness, a brief survey of discharges with neutral beam heating power up to $2 \mathrm{MW}$, featuring both low (L) and high (H) confinement modes, with double and single-null divertor geometry (at the bottom of the vacuum vessel) and with standard and reversed $\mathbf{B}_{T}$ directions were investigated. Otherwise identical $\mathrm{H}$ - and L-mode discharges, shown in Fig. 1, were analyzed in particular detail for comparative studies. The density was increased in a stepwise manner, as shown in Fig. 1, and the probe was inserted at various times, illustrated by two thick vertical lines, to assure coverage at several densities.

The principal measurements were made by a fast scanning probe array ${ }^{23}$ featuring five tips (Fig. 2, upper inset) that sense probe current $I$, ion saturation current $I_{\text {sat }}$ $=1 / 2 A_{p r} e n_{e} c_{s}=1 / 2 A_{p r} e n_{e}\left[k\left(T_{e}+T_{i}\right) / m_{i}\right]^{1 / 2}\left(T_{e}\right.$ and $T_{i}$ are the ion and electron temperatures, respectively, $A_{p r}$ is the tip area and $n_{e}$ is the electron density) and floating potential, $\Phi_{f}$. The measurements were utilized to infer the electron temperature, $T_{e}$, by using the harmonic method ${ }^{24}$ the electron density, $n_{e}$, by using the equation above (assuming $T_{e}$ $\left.=T_{i}\right)$ and the poloidal electric field, $E_{\theta}=\left(V_{f 1}-V_{f 2}\right) / \delta$, for probe tips separated a distance $\delta$; all with time resolution down to $1.0 \mu \mathrm{s}$. The calculation of $E_{\theta}$ assumes that the temperature and sheath drop are identical in both tips and that the intermittent objects are larger than the separation between the tips; the last assumption is justified later using beam emission spectroscopy (BES) data. The plasma potential $V_{p}$ was calculated as $V_{p} \sim \Phi_{f}+3.0 k T_{e}$. The probe scans horizontally from the outside wall in approximately $250 \mathrm{~ms}$ along the path indicated in Fig. 2. The Thomson scattering system, also shown in Fig. 2, provided independent $T_{e}$ and $n_{e}$ measurements at 12 locations separated by $2-3 \mathrm{~cm}$ every $12 \mathrm{~ms}$. The (BES) system, configured as a $5 \times 6$ fiber array as seen in Fig. 2 and inset, was located at the edge of the plasma in the midplane to provide fast $(1 \mu \mathrm{s})$ 2-D imaging of the density. The measurements, taken along the path of the probe insertion, are mapped onto the magnetic surfaces, displayed in Fig. 2, calculated by the toroidal equilibrium fitting code EFIT. ${ }^{25}$

\section{RESULTS: GENERAL CHARACTERIZATION OF THE INTERMITTENCY}

The probe signals for $I_{\mathrm{sat}}, V_{f}, E_{\theta}$ and $I_{\mathrm{sat}} E_{\theta}$ are shown in Fig. 3 for an interval $1 \mathrm{~ms}$ long for standard $\mathbf{B}_{T}$, L-mode 


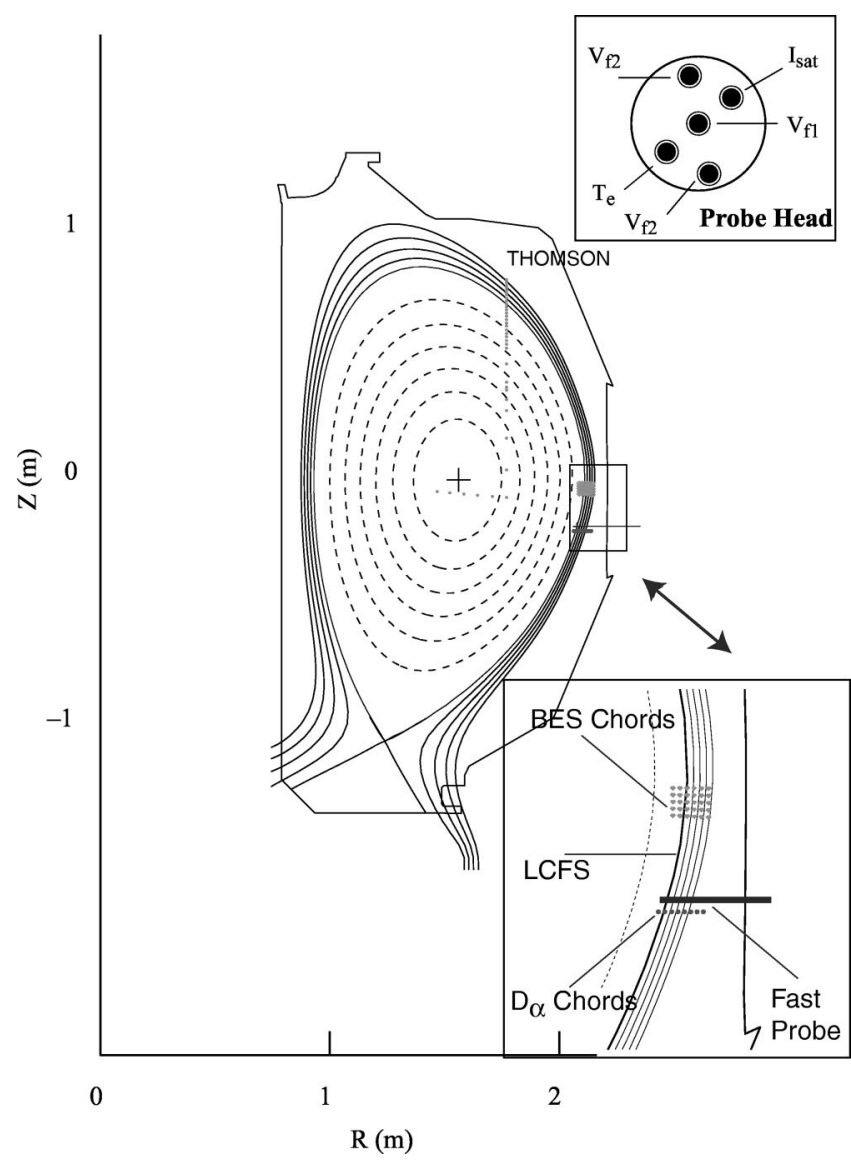

FIG. 2. Poloidal cut of DIII-D showing the magnetic geometry and some relevant diagnostics. Two insets show (top) the scanning probe tip geometry and (bottom) the BES diagnostic geometry. The Thomson scattering chords are indicated at the top.

discharges. The intermittent objects appear at a rate of $1-4$ $\times 10^{3} \mathrm{~s}^{-1}$. The product of $I_{\mathrm{sat}} E_{\theta}$ is used because it is straightforwardly calculated from raw probe data and is a quantity between radial particle $\left(n_{e} V_{r}=n_{e} E_{\theta} / B_{\phi}\right)$ and convective heat $\left(n_{e} V_{r} T_{e}=n_{e} T_{e} E_{\theta} / B_{\phi}\right)$ flux. Appreciable intermittency is evident and, although correlation of the large excursions can be observed by eye among all the traces, conditional averaging tools, introduced in the TEXT tokamak by Filippas ${ }^{15}$ and later in linear devices ${ }^{6}$ and other tokamaks, ${ }^{8}$ were used to quantify the observations. A $2.5 \times$ rms-level threshold was used to discriminate the intermittent events in $I_{\text {sat }}$, which were then binned for $100 \mu$ s about the maximum of each event, accumulated and then averaged. The existence of an event in $I_{\text {sat }}$ can be used as the condition to select time slices in the $E_{\theta}$ and $V_{f}$ signals at the same time slice.

The results of the conditional average on the $I_{\text {sat }}, E_{\theta}$ and $I_{\mathrm{sat}} E_{\theta}$ signals for three spatial locations in the SOL, namely, (a) near the separatrix, (b) $5 \mathrm{~cm}$ into the $\mathrm{SOL}$ and (c) $10 \mathrm{~cm}$ into the SOL, are shown in Fig. 4. Excellent correlation is found between events in the three signals and it can be noticed that (1) the $I_{\text {sat }}$ pulses are always positive, (2) the $E_{\theta}$ pulses are always positive and (3) the amplitudes of the $I_{\text {sat }}$ and $E_{\theta}$ events are quickly reduced (by a factor of $\sim 7$ ) away from the separatrix. Conditional averaging using various thresholds and utilizing $I_{\text {sat }}$ or $E_{\theta}$ as the primary signal yield equivalent results.

It is worth commenting that the conditional averaging technique with a window of $100 \mu$ s is applied to $20-40$ events, or a time series $2-5 \mathrm{~ms}$ long. The selected windows result on averaging over frequencies higher than those associated to the time scale of the binning window (or correspondingly, to structures that are smaller than the selected scale, which is in this case $\sim 2 \mathrm{~cm}$ ). Simultaneously, lower frequencies or larger spatial scales will appear as a dc offset in the averaged data. The time window used in the calculations presented in this article has been determined through trial and error.

The sign asymmetry that the intermittency conveys to the various signals (i.e., bursts are always positive in $I_{\text {sat }}$ ) affects their statistical characteristics and introduces a deviation from the Gaussian distribution that translates into a skewness ${ }^{13}$ of $\sim 1$ in the $I_{\text {sat }}$ signal, as shown in Fig. 5. Notice that the statistics for both the L-and $\mathrm{H}$-mode show significant skewness whereas a Gaussian distribution would feature a skewness of zero.

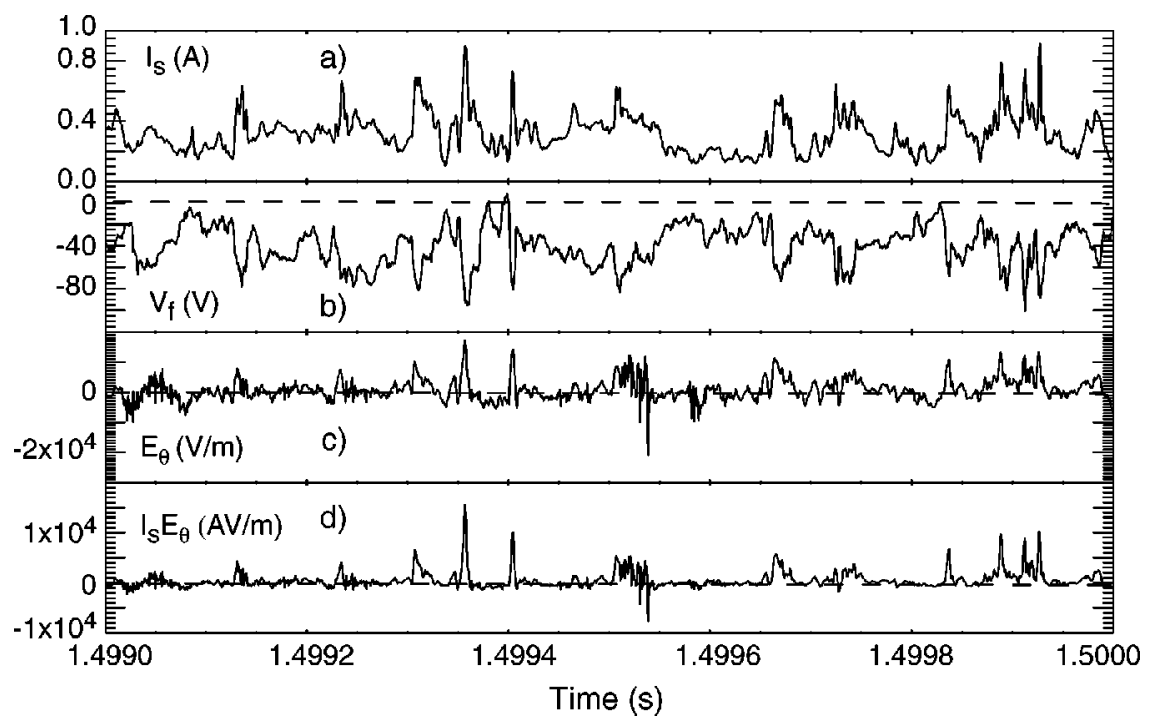

FIG. 3. Traces of $I_{\text {sat }}, V_{f}, E_{\theta}$, and $I_{\text {sat }} \times E_{\theta}$ are shown for a time interval of $1 \mathrm{~ms}$. Intermittency showing good cross-correlation is clearly visible in all the signals. 


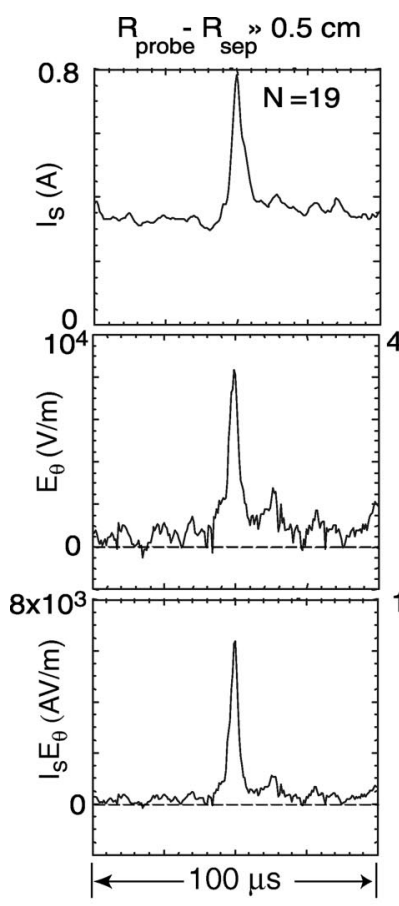

(a)

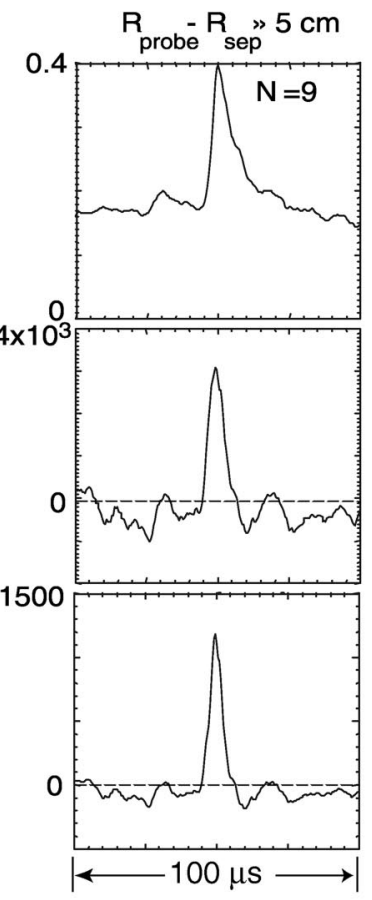

(b)

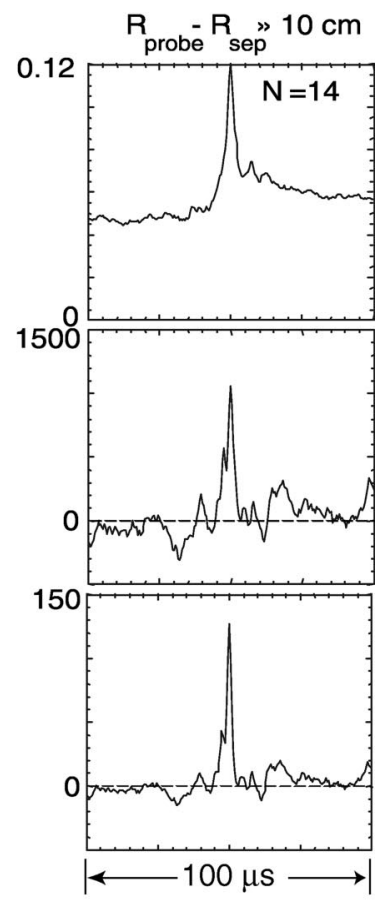

(c)
FIG. 4. Conditional averaging results of the $I_{\text {sat }}$ (top), $E_{\theta}$ (middle) and $I_{\text {sat }}$ $\times E_{\theta}$ signals for radial locations within (a) $0.5 \mathrm{~cm}$, (b) $5 \mathrm{~cm}$ and (c) 10 $\mathrm{cm}$ of the LCFS, showing the intermittent feature and its amplitude decaying with radius. The number of events found in the $5 \mathrm{~ms}$ interval, $N$, is noted.
The data obtained insofar indicate that discrete, intermittent plasma objects (IPOs) are traveling past the probe tips. The IPO's radial convection velocity, $\mathbf{v}_{E}=\mathbf{E} \times \mathbf{B} / B^{2}$, can be easily calculated from the measured $E_{\theta}$ and the local value of $B_{t}$ at the measurement location $(\sim 15 \mathrm{kG})$. The validity of this measurement depends on $T_{e}$ being similar at both poloidally separated tips, which is equivalent to stating that the gradient scale length of the IPO's internal field is greater than the distance between tips. As we will show later and support with BES measurements, the IPOs are $\sim 3 \times 3 \mathrm{~cm}^{2}$ in size and thus much larger than the $3 \mathrm{~mm}$ distance between the tips. The assumption here is that the various quantities associated with the objects (in this case density and temperature) are of similar spatial scale. Once their radial velocity is estimated, their radial extent can be calculated from $\delta r$ $=V_{r} \Delta t$ where $\Delta t$ is the duration of the bursts. The calculations, shown in Table I, show that the IPOs begin with a velocity of $\sim 2600 \mathrm{~m} / \mathrm{s}$ at the separatrix and slow down to

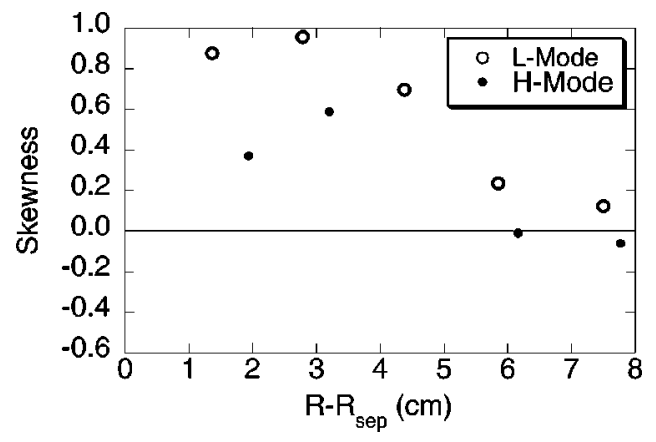

FIG. 5. The skewness of the $I_{\text {sat }}$ signal is shown for L-mode (open circles) and $\mathrm{H}$-mode (solid circles) discharge conditions. Both show marked deviation from Gaussian behavior. about $330 \mathrm{~m} / \mathrm{s}$ at the wall $(10 \mathrm{~cm}$ farther $)$ and their size starts as roughly $3-4 \mathrm{~cm}$, shrinking to $0.5 \mathrm{~cm}$ near the wall.

The poloidal velocity of the IPOs can be calculated using two techniques, namely time-lag correlation and timedelay of conditionally averaged signals from the two poloidally separated probe tips. A comparison of the aforementioned techniques to the $E_{r} \times B$ velocity, is shown in Fig. 6. All techniques show a fast decay of the IPO's poloidal velocity away from the LCFS into the SOL; however, the velocity inferred from the time-delay technique, $V_{\text {del }}$, is much higher than those inferred from the correlation technique, $V_{\text {corr }}$, and the $E_{r} \times B$ velocity. Furthermore, the IPO's poloidal velocity inferred from the BES diagnostic (marked as a large open circle in Fig. 6) agrees with the time-delay technique. Although the issue is still being debated, the data seem to indicate that the IPOs move faster than the background plasma, which is the velocity that $E_{r}$ $\times B$ and time-lag correlation tend to represent. The data are consistent with a poloidal object size of about $\sim 3 \mathrm{~cm}$.

More can be learned about the IPOs by studying their temperature distribution. A fast $T_{e}$ diagnostic ${ }^{26,24}$ with bandwidth of $200 \mathrm{kHz}$, and thus able to resolve the temperature inside the structures, is used. The results from conditional averaging of the $T_{e}$ trace, shown in Fig. 7, indicate that the

TABLE I. Summary of IPO parameters as a function of the distance from the separatrix $\left(R_{\text {probe }}-R_{\text {sep }}\right)$. The IPOs duration, $\Delta t$, and internal poloidal field, $E_{\theta}$, allow the calculation of their radial velocity, $V_{r}$, and size, $\Delta_{r}$.

\begin{tabular}{cccccc}
\hline \hline & $R_{\text {probe }}-R_{\text {sep }}$ & $\Delta t(\mathrm{~s})$ & $E_{\theta}(\mathrm{V} / \mathrm{m})$ & $V_{r}(\mathrm{~m} / \mathrm{s})$ & $\Delta r(\mathrm{~cm})$ \\
\hline LCFS & 0.5 & $1.5 \times 10^{-5}$ & 4000 & 2660 & 3.9 \\
& 5 & $2.0 \times 10^{-5}$ & 1500 & 1000 & 2.0 \\
Wall & 10 & $1.5 \times 10^{-5}$ & 500 & 330 & 0.5 \\
\hline \hline
\end{tabular}




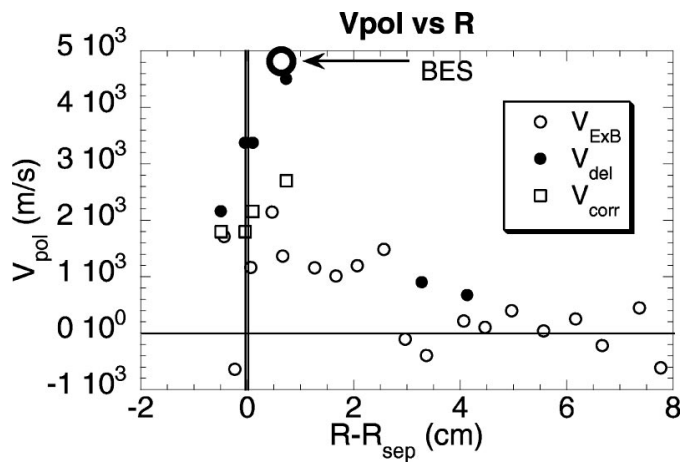

FIG. 6. Intermittency's poloidal velocity estimates by using time-delay techniques (solid circles) and time-lag correlation (open squares). The data are compared to the $E_{r} \times B$ velocity and one point from BES (large open circle).

temperature in the IPOs [Fig. 7(a)] is $150 \mathrm{eV}$, nearly three times that of the background $(50 \mathrm{eV})$, and their potential [Fig. 7(d)], calculated as $V_{p} \approx V_{f}+3 k T_{e}\left[V_{f}\right.$ shown in Fig. $7(\mathrm{c})$ ], is $400 \mathrm{~V}$ and positive with respect to the surrounding plasma $(120 \mathrm{~V})$, suggesting that a predominant electron loss occurs at the IPO boundaries. Furthermore, the burst in plasma potential in Fig. 7(d) indicates that the IPOs (with dimensions of roughly $3 \times 3 \mathrm{~cm}^{2}$ in the poloidal plane) contain a radial electric field, $E_{r}=-\nabla V_{p}$, that can be estimated as $E_{r} \approx \Delta V_{p} / \delta r=300 / 1.5(\mathrm{~V} / \mathrm{cm})$ and results in internal rotation at a $V_{\theta^{\prime}}=E_{r^{\prime}} / B_{t}$ speed of $1.3 \times 10^{4} \mathrm{~m} / \mathrm{s}$. In the above measurements, the only assumption is that we can resolve the temperature in these objects fast enough and that the plasma potential can be calculated as $V_{p} \approx V_{f}+3 k T_{e}$.

\section{RESULTS: EVIDENCE FROM OTHER DIAGNOSTICS}

The data from the probes indicates that events greater than two times the rms level of the $I_{\text {sat }}$ or $T_{e}$ signals are not uncommon in these discharges, and therefore should be apparent in the signals from other diagnostics such Thomson scattering and BES. ${ }^{27,28}$
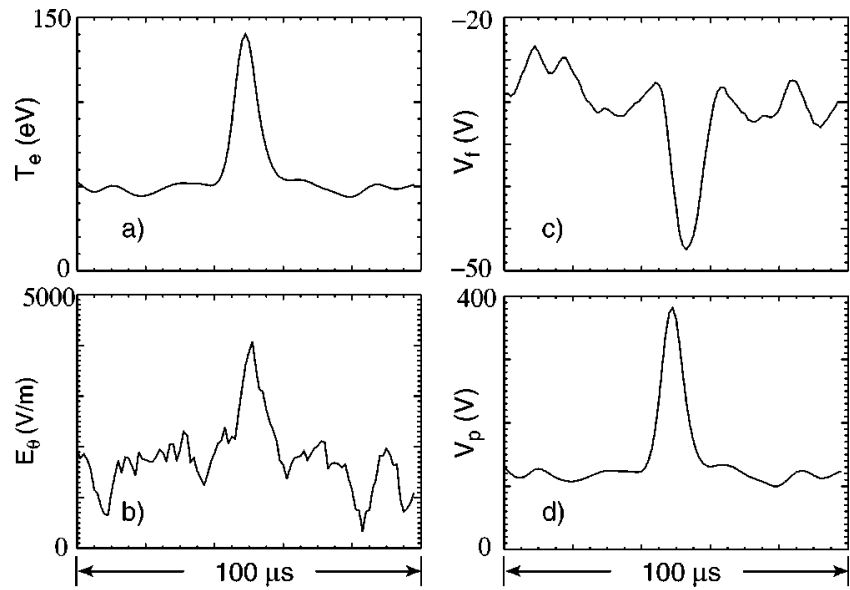

FIG. 7. Conditional average results for (a) $T_{e}$, (b) $E_{\theta}$, (c) $V_{f}$, and (d) $V_{p}$ showing that (a) the plasma structures are charged positively, (b) contain and convect substantial amounts of energy and (c) a positive radial electric field exists inside them.
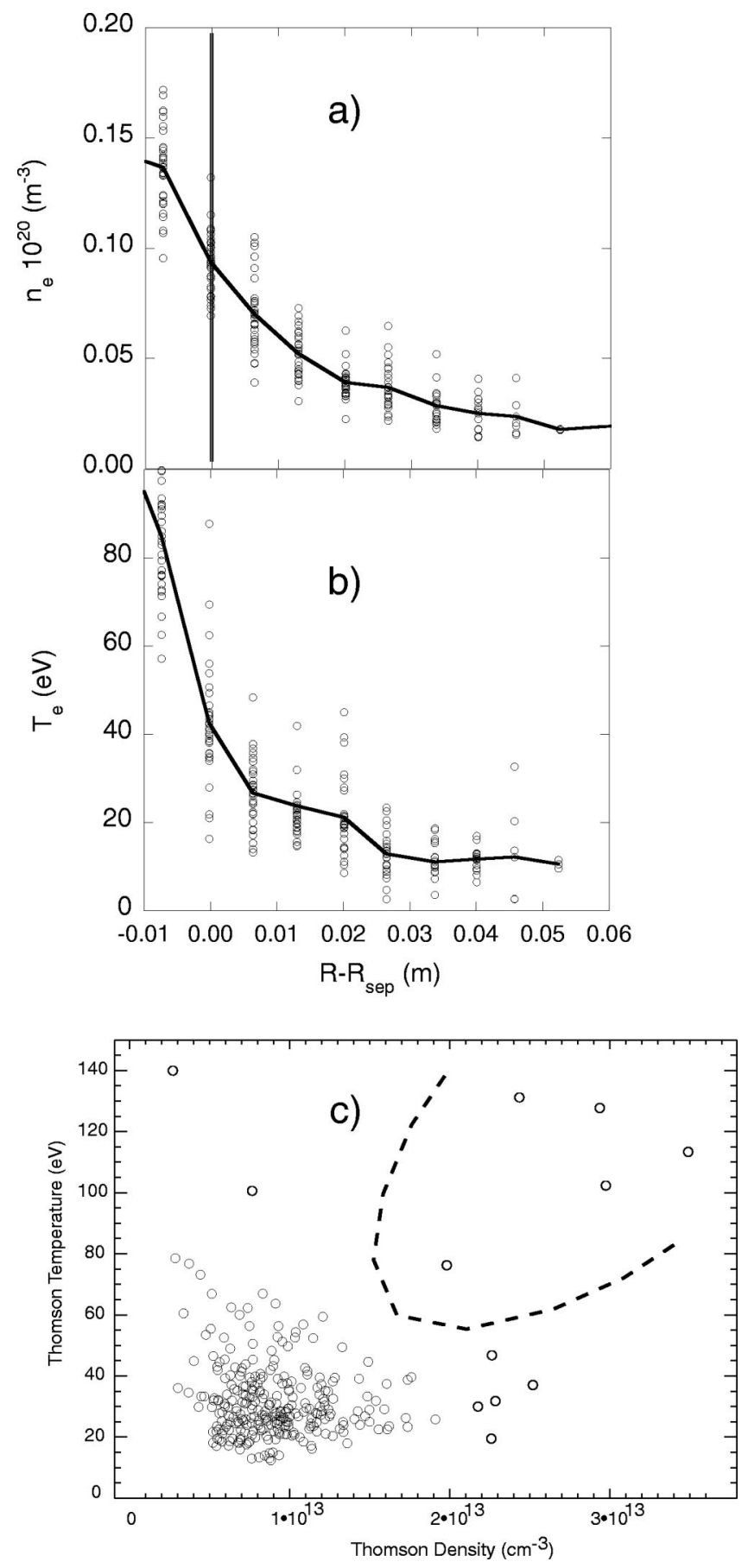

FIG. 8. (a) and (b) $T_{e}$ and $n_{e}$ from Thomson scattering data accumulated every $12 \mathrm{~ms}$ for $500 \mathrm{~ms}$ and compared to the average showing that fluctuations are high, reflecting the intermittency. (c) Plot of $T_{e}$ and $n_{e}$ from Thomson scattering data accumulated for $500 \mathrm{~ms}$ in L-mode indicating that there are points featuring both with high density and temperature in support for probe data. These points are beyond the dashed line.

The Thomson scattering diagnostic at DIII- $\mathrm{D}^{29}$ probes the plasma for $25 \mathrm{~ns}$ every $12 \mathrm{~ms}$, clearly insufficient to properly sample the intermittency but sufficient to provide a rough comparison to the probe data. Thomson scattering density and temperature data accumulated for $500 \mathrm{~ms}$ in an L-mode discharge (to avoid the complication of ELMS) and superimposed over the average are shown in Figs. 8(a) and $8(\mathrm{~b})$, featuring large temperature $(\sim 80 \%)$ and density 


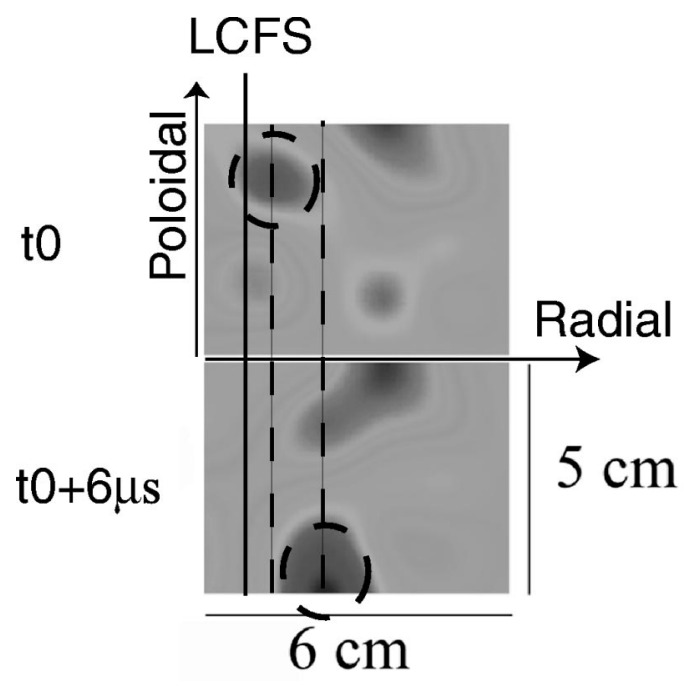

FIG. 9. Two frames from BES showing 2-D density plots. There is a time difference of $6 \mu$ s between frames. A particular structure is marked with a dashed circle, and shown in both frames, clearly highlighting poloidal and radial motion.

$(\sim 40 \%)$ fluctuation levels. Notice that the relative fluctuation levels increase with radius. The excursions in temperature and density are correlated in time, as shown in Fig. 8(c) for a Thomson channel $1 \mathrm{~cm}$ into the SOL, supporting the paradigm of high density and temperature IPOs buffeting the probe tips.

Further support is obtained from the BES diagnostic, which consists of a fiber array that covers a $6 \times 5 \mathrm{~cm}^{2}$ region at the edge plasma as previously shown in Fig. 2. Two frames, where the LCFS is indicated by a vertical solid line, taken at an arbitrary time $t_{0}$ and $6 \mu \mathrm{s}$ later $\left(t_{0}+6 \mu \mathrm{s}\right)$ are shown in Fig. 9. A positive density feature is marked by a dashed circle in both frames and followed through the six frames. The center of the feature is indicated by vertical dashed lines. Notice that the object has a spatial extent of roughly $2 \times 2 \mathrm{~cm}^{2}$. From the two frames it is clear that the object is moving poloidally and radially with speeds that can be easily estimated at $V_{\theta}=5 \mathrm{~km} / \mathrm{s}$ and $V_{r}=1500 \mathrm{~m} / \mathrm{s}$, values which compare well to probe data.

\section{RESULTS: L-H MODE COMPARISON}

Intermittency data from discharges in L- and H-mode that are otherwise identical, as seen in Fig. 1, were analyzed with conditional averaging. The results for density, showing the CFOs, are shown in Fig. 10 by superimposing traces from various radii and indicating (arrow) the direction of increasing radius. Three properties of the data are immediately apparent: (1) The density excursion is two to three times the background density, (2) the amplitude of the pulses for similar radius is much larger for L-mode conditions than for H-mode conditions, and (3) the amplitude of the pulses decays rapidly with radius.

Evaluation of the total particle flux convected by the IPOs is of utmost importance to assess their relevance to the radial transport. The flux can be calculated as $\Gamma_{\mathrm{Int}}=n_{e}\left(E_{\theta}\right.$ $\times B / B^{2}$ ) and the result is shown in Fig. 11 for L- and

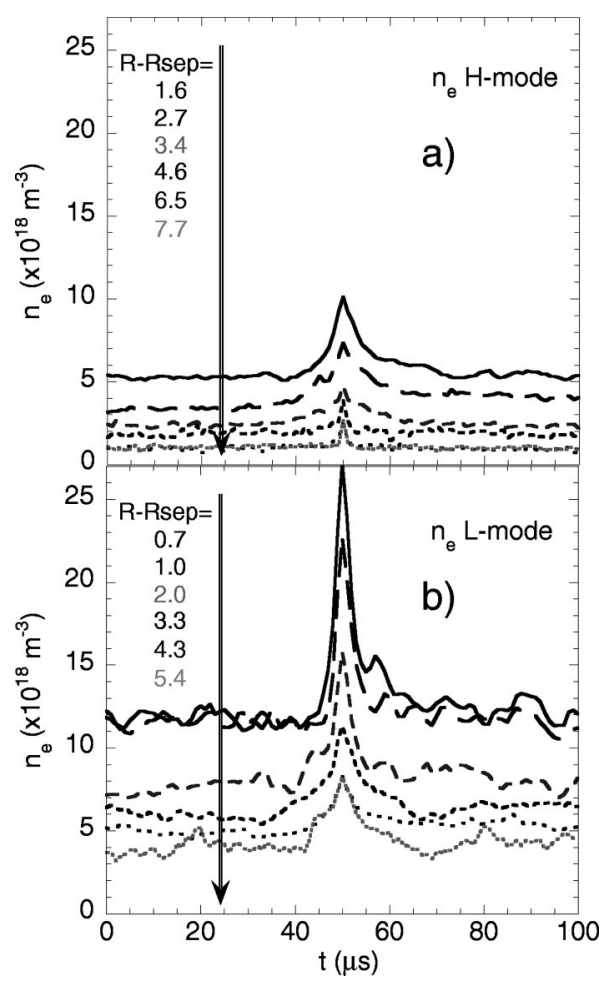

FIG. 10. Conditional averaging results showing the density pulses for Land $\mathrm{H}$-mode conditions. Data from different radii are superimposed, those from the LCFS at the top and those from the far SOL at the bottom, as the arrows indicate. The corresponding radii are indicated. Density pulses in L-mode are larger than in H-mode.

$\mathrm{H}$-mode conditions and in the same scale. Three properties are again quite apparent: (1) The flux excursion is large and nearly five times the background, (2) the flux for similar radius is much larger for $\mathrm{L}$-mode conditions than for $\mathrm{H}$-mode conditions, and (3) the flux decays rapidly with radius.

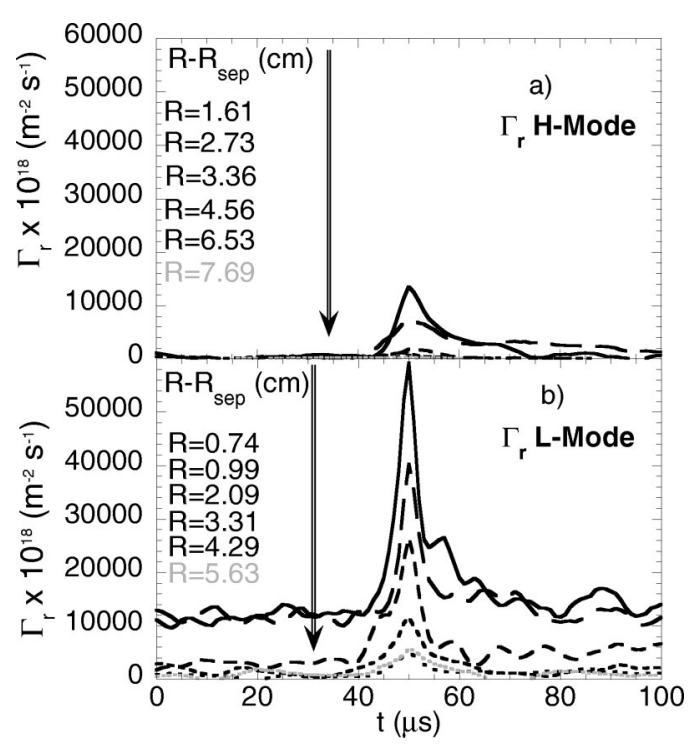

FIG. 11. Conditional averaging results showing the intermittent particle flux for L- and H-mode conditions. Data from different radii are superimposed, those from the LCFS at the top and those from the far SOL at the bottom, as the arrows indicate. The corresponding radii are indicated. Particle flux in $\mathrm{L}$-mode is larger than in H-mode. 


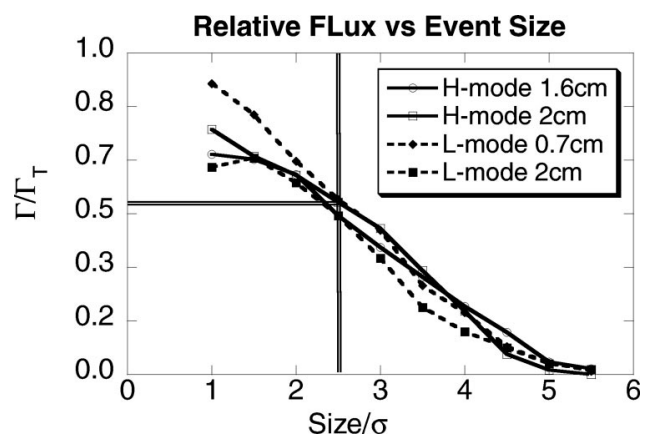

FIG. 12. Ratio of the intermittent particle flux to the total $E \times B$ flux as a function of event size normalized to rms levels. Events greater than $2.5 \times$ rms carry $50 \%$ of the transport.

The relevance of IPOs to radial transport in the SOL is obtained in $\mathrm{L}$ - and $\mathrm{H}$-mode at various radii by calculating $\Gamma_{\text {Int }} / \Gamma_{\text {Total }}=\left\langle I_{\text {sat }} E_{\theta}\right\rangle_{\text {Intermittent }} /\left\langle I_{\text {sat }} E_{\theta}\right\rangle_{\text {Total }}$ versus eventamplitude thresholds expressed as a multiplier of the rms level. That is, each point shows the fraction of transport arrived by events above the rms multiplier shown in the abscissa. The result, shown in Fig. 12, indicates that (a) intermittent events above the $2.5 \times \mathrm{rms}$ level are responsible for $\sim 50 \%$ of the transport at all radii, (b) there is a continuum of event sizes, and (c) although the relative relevance of intermittency is unchanged with radius, its absolute amplitude decays rapidly with radius due to the decay of the structures themselves.

It is important to notice that the intermittency under study here, residing in the $1-200 \mathrm{kHz}$ band, is part of the usual $E \times B$ electrostatic turbulent particle flux, $\widetilde{\Gamma}_{r}$, that depends on the rms value of the density, $\left\langle\widetilde{n}_{e}^{2}\right\rangle^{1 / 2}$, poloidal field, $\left\langle\widetilde{E}_{\theta}^{2}\right\rangle^{1 / 2}$ and their cross-correlation. $\widetilde{\Gamma}_{r}$ is plotted in Fig. 13 for the same L- and $\mathrm{H}$-mode conditions, indicating a large reduction of the total $E \times B$ transport during $\mathrm{H}$-mode and it is important to consider these data in the framework of Fig. 12 showing that the intermittent structures fraction of the total transport is constant with radius and that there is a con-

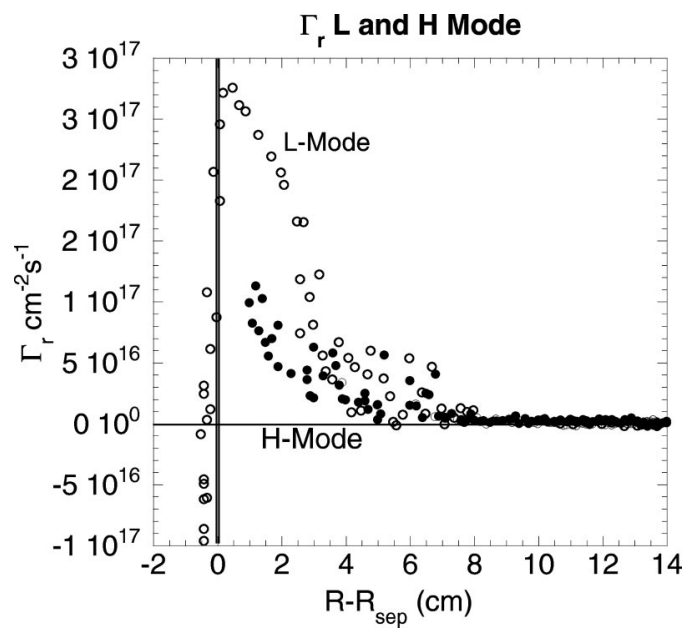

FIG. 13. Profiles of the radial broadband $E \times B$ flux for L- and H-mode conditions. Flux in L-mode is four to five times greater than in $\mathrm{H}$-mode.

Thus $E \times B$ transport, including intermittency, is suppressed.

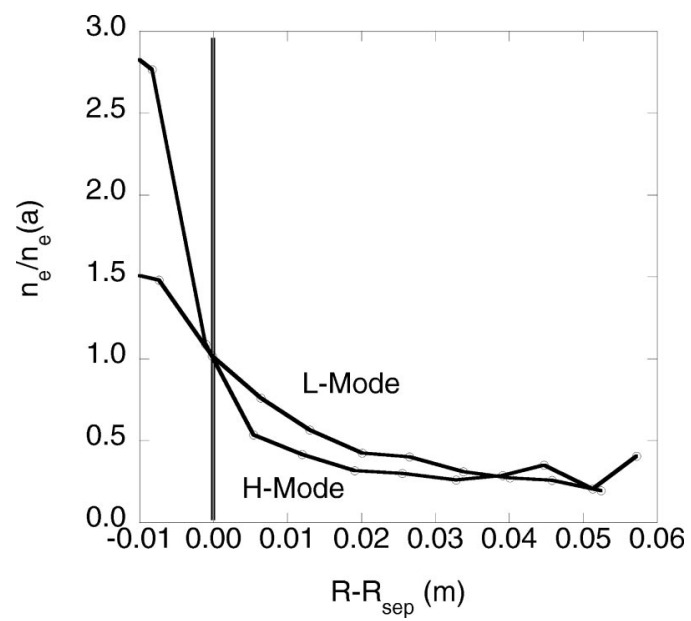

FIG. 14. Thomson density profiles for L- and H-mode discharges. After a fast drop at the LCFS, the profiles flatten up to the wall.

tinuum of event sizes. The results strongly suggest that the intermittency is part of the turbulence. It can therefore be stated that the intermittency, and its response to sheared electric fields that decorrelate turbulent transport resulting in reduced transport and enhanced confinement conditions, has been indirectly characterized extensively in many devices such as CCT, ${ }^{30,31}$ PBX-M, ${ }^{32,33}$ DIII-D, ${ }^{34}$ TEXT, ${ }^{35,36}$ and TEXTOR. ${ }^{37,38}$

Density profiles from Thomson scattering in the SOL, normalized to the value at the LCFS, are shown for L- and $\mathrm{H}$-mode discharges in Fig. 14, showing that there is a clear difference in the profile decay length near the LCFS $(0.5 \mathrm{~cm}$ $\mathrm{H}$-mode to 1.3 in L-mode), but that in the far SOL both profiles are quite flat and the particle density is significant. It is problematic to invoke diffusion as the radial transport mechanism in the far SOL if the density gradient is zero and thus convection needs to be called upon, possibly in the form of intermittent events. However, the quick reduction in the amplitude of the bursts and concomitant fast decay of the density profiles is not in contradiction with a strong particle sink in the divertor for the conditions considered.

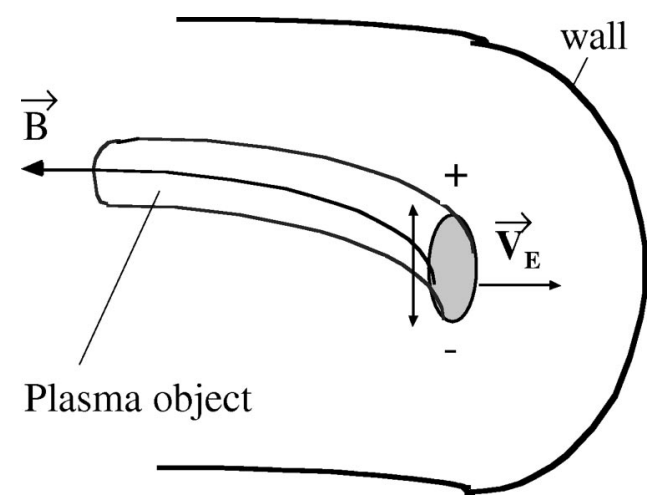

FIG. 15. Plasma filament polarization and associated drift result in outward motion of plasma structure in the tokamak boundary. The drift velocity of the object is $V_{b}=V_{E} \approx c E / B$. 

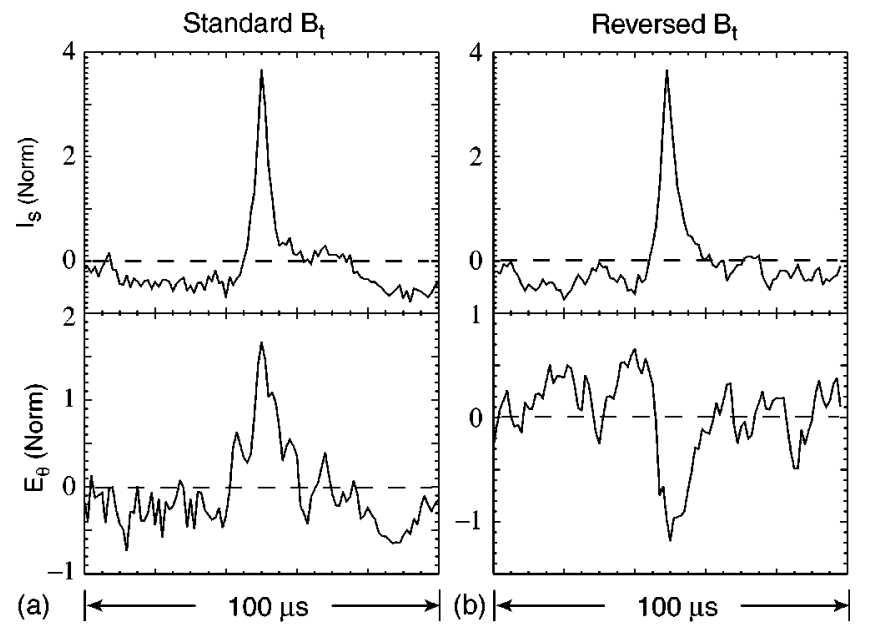

(a) $\longleftarrow 100 \mu \mathrm{s}$
FIG. 16. Conditional average of the $I_{\text {sat }}$ and $E_{\theta}$ signals for (a) standard $B_{T}$ : events in $E_{\theta}$ are positive and (b) reserved $B_{T}$ : events in $E_{\theta}$ are negative. The results support the $\nabla B$ polarization mechanism.

\section{TEST OF SIMPLE DYNAMICAL MODEL}

A simple model can be built, ${ }^{39}$ as illustrated in Fig. 15, for the dynamics of the IPOs under the assumption that the radial motion is based on the polarization caused by $\nabla B$ and magnetic field curvature ${ }^{40}$ and associated $\vec{E} \times \vec{B}_{T}$ drift. An estimate can then be made of both the velocity of the CFOs and the radial distances they can propagate before they disappear due to parallel plasma particle loss. If this hypothesis is correct, reversal of $\mathbf{B}$ would result in an inversion of $E_{\theta}$ while maintaining the outwardly radial $\mathbf{E} \times \mathbf{B} / B^{2}$ velocity. Discharges that feature opposite signs of $B_{T}$, but are otherwise identical, were investigated and the result of conditional averaging is shown in Fig. 16. A reversal in $E_{\theta}$ is clearly correlated to a reversal in $B_{T}$, always resulting in outwardlydirected transport, supporting, although not proving, the $\nabla B$ polarization mechanism.

\section{CONCLUSIONS}

Intermittency in the boundary of the DIII-D tokamak during L- and $\mathrm{H}$-mode discharges has been analyzed using conditional averaging and the associated radial transport interpreted in the framework of $E \times B_{T}$ driven structures. The results show that IPOs exist in both L- and H-mode featuring higher density, temperature and potential than the surrounding plasma and transporting particles and heat into the far SOL by convection. The IPOs slow down quickly and lose particles as they move out into the far SOL. Although the IPOs carry more particles in L-mode, their relative contribution to particle transport is evaluated at $\sim 50 \%$ of the total $E \times B$ transport in all the conditions considered. Thus, the CFOs seem to be an integral part of the background turbulence. Therefore, detailed research to investigate the origins of the structures and conditions under which they appear is paramount.

\section{ACKNOWLEDGMENTS}

Discussions with K. Burrell are acknowledged.
This research was supported by the U.S. Department of Energy under Contract Nos. DE-AC03-99ER54463 and W-7405-ENG-48, and Grant No. DE-FG03-95ER54294.

${ }^{1}$ P. Stangeby, The Plasma Boundary of Magnetic Fusion Devices (IOP, Bristol, 2000), p. 46.

${ }^{2}$ J. Watkins, R. Moyer, J. W. Cuthbertson et al., Rev. Sci. Instrum. 68, 373 (1997).

${ }^{3}$ J. Boedo, D. Gray, L. Chousal et al., Rev. Sci. Instrum. 69, 2663 (1998).

${ }^{4}$ B. LaBombard, M. V. Umansky, R. L. Boivin et al., Nucl. Fusion 40, 2041 (2000).

${ }^{5}$ M. R. Wade, W. P. West, R. D. Wood et al., J. Nucl. Mater. 266-269, 44 (1999).

${ }^{6}$ A. H. Nielsen, H. L. Pecsell, and J. Juul Rasmussen, Phys. Plasmas 3, 1530 (1996).

${ }^{7}$ R. D. Lehmer, Ph.D. thesis, University of California Los Angeles, UCSDENG-032, 1996.

${ }^{8}$ E. Sanchez, C. Hidalgo, C. Riccardi et al., Phys. Plasmas 7, 1408 (2000).

${ }^{9}$ B. K. Joseph, R. Jha, P. K. Kaw et al., Phys. Plasmas 4, 4292 (1997).

${ }^{10}$ R. A. Moyer, R. D. Lehmer, T. E. Evans et al., Plasma Phys. Controlled Fusion 38, 1273 (1996).

${ }^{11}$ R. Jha and Y. C. Saxena, Phys. Plasmas 3, 2979 (1996).

${ }^{12}$ B. Carreras, V. E. Lynch, D. E. Newman et al., Phys. Plasmas 7, 3278 (2000).

${ }^{13}$ W. Press, S. Teukolsky, W. Vettering, and B. Flannery, Numerical Recipes in Fortran (Cambridge University Press, Cambridge, 1986), p. 606.

${ }^{14}$ E. Sanchez, C. Hidalgo, D. Lopez-Bruna et al., Phys. Plasmas 7, 1408 (2000).

${ }^{15}$ A. V. Filippas, R. D. Bengtson, G. X. Li et al., Phys. Plasmas 2, 839 (1995).

${ }^{16}$ J. Soteckel, M. V. Heller, J. Petrzilka et al., Phys. Plasmas 6, 846 (1999).

${ }^{17}$ I. Garcia-Cortes, R. Balbin, A. Loarte et al., Plasma Phys. Controlled Fusion 42, 389 (2000).

${ }^{18}$ G. Antar, S. Krasheninnikov, P. Devynck et al., Phys. Rev. Lett. 87, 065001 (2001).

${ }^{19}$ G. Antar, P. Devynck, X. Garbet, and S. Luckhardt, Phys. Plasmas 8, 1612 (2001).

${ }^{20}$ S. J. Z. and S. S. Medley, Phys. Fluids B 1, 2058 (1989).

${ }^{21}$ R. Maqueda, G. Wurden, S. Zweben et al., Rev. Sci. Instrum. 72, 931 (2001).

${ }^{22}$ J. C. Luxon and L. G. Davis, Fusion Technol. 8, Part 2A, 441 (1985).

${ }^{23}$ J. Watkins, J. Salmonson, R. Moyer et al., Rev. Sci. Instrum. 63, 4728 (1992).

${ }^{24}$ J. A. Boedo, D. Gray, M. Schaffer et al., Rev. Sci. Instrum. 70, 2997 (1999).

${ }^{25}$ L. L. Lao, H. St. John, R. O. Stambaugh et al., Nucl. Fusion 25, 1611 (1985).

${ }^{26}$ D. Rudakov, J. Boedo, R. Moyer et al., Rev. Sci. Instrum. 72, 453 (2001).

${ }^{27}$ G. McKee, R. Ashley, R. Durst et al., Rev. Sci. Instrum. 70, 913 (1999).

${ }^{28}$ C. Fenzi, R. J. Fonck, M. Jakubowski, and G. R. McKee, Rev. Sci. Instrum. 72, 988 (2001).

${ }^{29}$ T. N. Carlstrom, G. L. Campbell, J. C. DeBoo et al., Rev. Sci. Instrum. 63, 4901 (1992)

${ }^{30}$ G. R. Tynan, J. Liberati, P. Pribyl, R. J. Taylor, and B. Wells, Plasma Phys. Controlled Fusion 38, 1301 (1996).

${ }^{31}$ G. R. Tynan, L. Schmitz, R. W. Conn, R. Doerner, and R. Lehmer, Phys. Rev. Lett. 68, 3032 (1992).

${ }^{32}$ G. Tynan, L. Schmitz, L. Blush et al., Plasma Phys. Controlled Fusion 36, Suppl. 7A, 285 (1994).

${ }^{33}$ G. R. Tynan, L. Schmitz, H. Kugel et al., Phys. Plasmas 1, 3301 (1994).

${ }^{34}$ R. A. Moyer, K. Burrell, T. Carlstrom et al., Phys. Plasmas 2, 2397 (1995).

${ }^{35}$ T. Rhodes, C. P. Ritz, and R. D. Bengtson, Nucl. Fusion 33, 1147 (1993).

${ }^{36}$ H. Tsui, A. Wooton, R. Bengtson et al., Plasma Phys. Controlled Fusion 2, 673 (1993).

${ }^{37}$ J. Boedo, D. Gray, S. Jachmich et al., Nucl. Fusion 40, 209 (2000).

${ }^{38}$ J. Boedo, P. W. Terry, D. Gray et al., Phys. Rev. Lett. 84, 2630 (2000).

${ }^{39}$ A. V. Nedospasov, V. G. Petrov, and G. N. Fidel'man, Nucl. Fusion 25, 21 (1985).

${ }^{40}$ S. Krasheninnikov, Phys. Lett. A 283, 368 (2001). 